\title{
The Dubrava Model: A Novel Approach
}

\author{
Peter Reilly ${ }^{1}$ \\ ${ }^{1}$ School of Health and Medical Sciences, University of Adelaide, \\ Adelaide, South Australia
}

\section{J Neurosci Rural Pract 2019;10:389}

The authors present a plan for the management of patients with severe head injury who are admitted directly to a rural hospital without a resident neurosurgeon. According to this plan, a neurosurgeon on call at the major urban hospital travels by road to the rural hospital to perform the essential emergency operation. ${ }^{1}$

The Dubrava model has been functioning for 5 years. So far 320 emergency operations have been performed in the four rural hospitals involved in the scheme.

The authors address an important issue, namely that many trauma and guideline systems developed in high-income countries are not universally applicable. Trauma systems aim to provide rapid, expert treatment in the safest way. The structure of a trauma system needs to consider the distribution of expertise, geographical factors, and available retrieval services.

The Dubrava model aims to reduce the time to definitive surgical treatment by bringing the neurosurgeon to the patient and to avoid the risks inherent in transporting the patient to the city hospital.

If their model can reduce risk and provide rapid expert care, then it is to be applauded.

A critical matter in acute head injury care is time to definitive treatment. The authors acknowledge this in their discussion. They suggest that in their region, it is probably faster for a neurosurgeon to travel to the regional hospital than for an ambulance to transport the patient to the neurosurgeon, a method that involves additional and potentially risky transfers.

A trauma system needs to include care of patients with nonsurgical head injury and those needing intensive care unit (ICU) care postoperatively. In setting up a "Dubrava model," these factors must be incorporated. Are there ICU beds in the
Address for correspondence Peter Reilly, MD, BS, BMedSc (hons), FRACS, FFPMANZCA, School of Health and Medical Sciences, University of Adelaide, 81 Queen St Norwood South Australia 5067, Australia (e-mail: p.reilly@adelaide.edu.au).

regional hospital able to manage and monitor patients with severe traumatic brain injury (TBI), either postsurgical or nonsurgical? Should the TBI patients stay in the general hospitals or will they need to be transferred to the major hospital postsurgery? If they remain in the regional hospital, does the neurosurgical service remain involved in the care? Where will rehabilitation take place?

The authors list the number of intracranial hematomas treated.

To fully assess the benefits of this trauma system, it will be important to audit the times from accident to admission, times to definitive surgery, postoperative management, discharge and follow-up details, and outcomes and to reference these data to admissions to the major hospital.

The authors recommend the Dubrava model as a significant advance in the management of acute neurotrauma in their region. This may well be so, and they deserve acknowledgment for developing and maintaining this system. The model may be relevant to other regions after carefully considering the risks and benefits which might apply in that region.

\author{
Funding \\ None. \\ Conflict of Interest \\ None declared.
}

\section{Reference}

1 Orešković D, Raguž M, Almahariq F, et al. The Dubrava model - A novel approach in treating acutely neurotraumatized patients in rural areas: a proposal for management. J Neurosci Rural Pract 2019;10(3):446-451 